PHYSICAL REVIEW E 76, 011605 (2007)

\title{
Contact lines for fluid surface adhesion
}

\author{
Markus Deserno and Martin Michael Müller \\ Max-Planck-Institut für Polymerforschung, Ackermannweg 10, 55128 Mainz, Germany
}

Jemal Guven

Instituto de Ciencias Nucleares, Universidad Nacional Autónoma de México, Apdo. Postal 70-543, 04510 México D.F., Mexico

(Received 1 March 2007; published 26 July 2007)

\begin{abstract}
When a fluid surface adheres to a substrate, the location of the contact line adjusts in order to minimize the overall energy. This implies boundary conditions which depend on the characteristic surface deformation energies. We develop a general geometrical framework within which these conditions can be derived in a completely systematic way. We treat both adhesion to a rigid substrate and adhesion between two fluid surfaces, and illustrate our general results for several important Hamiltonians involving both curvature and curvature gradients. Some of these have previously been studied using very different techniques. With the exception of capillary phenomena, the Hamiltonian will not only be sensitive to boundary translations, but may also respond to changes in slope and even in curvature. The functional form of the additional contributions will follow readily from our treatment. We will show that the boundary conditions describing adhesion between two fluid surfaces express the balance of stresses and torques, as one would expect. At a rigid substrate, however, this simple identification will generally fail. This is because local rotations of the surface normal will be entirely "enslaved" to translations on the substrate. As a consequence, stresses and torques enter a single balance condition and cannot be disentangled.
\end{abstract}

DOI: 10.1103/PhysRevE.76.011605

PACS number(s): 68.03.Cd, 87.16.Dg, 02.40.Hw

\section{INTRODUCTION}

When surfaces occur in physical systems, the energy associated with them is often completely described by a Hamiltonian that depends only on the surface geometry. The easiest and the best known examples involve capillary phenomena $[1,2]$, in which the shape of a liquid-fluid interface is the geometry minimizing its area. The same physics underlies the behavior of soap films. Higher-order surface properties, notably its curvature, play a role in the description of fluid lipid membranes or microemulsions [3,4], and even higher derivatives have been implicated in the occurrence of certain corrugated membrane phases [5]. In all these cases the shape of the surface follows from minimizing the surface Hamiltonian, a variational problem. The corresponding EulerLagrange differential equations are known as the shape equations.

However, such surfaces are generally not isolated but rather in contact with something else. Water droplets or lipid membrane vesicles may rest on a substrate, and this generally influences their shape quite strongly. For instance, water droplets on hydrophilic substrates (e.g., clean glass) resemble flat contact lenses, while on very hydrophobic substrates (e.g., Teflon) they are almost completely spherical. When gravity can be neglected [6], the shape equation dictates a constant mean curvature surface in both cases (in fact, a spherical cap), but the contact angle at the three-phase line where water and substrate meet is different for the two different substrates.

In the majority of cases the spatial extension of the surface being studied exceeds the range of the interaction between it and the substrate by a large amount. For instance, van der Waals forces, hydrophobic interactions, or (screened) electrostatic forces typically extend over several nanometers, while the extensions of vesicles or droplets can be microns or even millimeters. Under these conditions the interaction is well approximated by a contact energy-i.e., an energy per unit area, $w$-liberated when the surface makes contact with the substrate. It is this adhesion energy, together with the energy parameters characterizing the contacting surfaces, which determines the boundary conditions holding at the contact line. In the case of capillary phenomena, for example, the ratio between adhesion energy $w$ and surface tension $\sigma$ determines the contact angle $\vartheta$ between liquid and substrate surface by means of the well-known Young-Dupré equation $[1,2,7]$

$$
\frac{w}{\sigma}=1+\cos \vartheta
$$

The "standard" derivation of this equation involves a balance of tangential forces at the contact line. Yet, despite its very intuitive nature, the requirement of surface stress balance does not yield the correct condition for more complicated surface Hamiltonians, even if the concept of surface stress is generalized properly. Higher-order Hamiltonians give rise to additional energy contributions when the contact line is varied. It is the purpose of the present article to show how these contributions can be accounted for in a systematic and parametrization-free way, and without assuming any additional symmetries (such as axisymmetry or translation symmetry along the contact line). We study adhesion to rigid substrates as well as to deformable surfaces also characterized by a surface Hamiltonian. Our presentation generalizes and strongly simplifies the analysis previously given in Ref. [8]. 


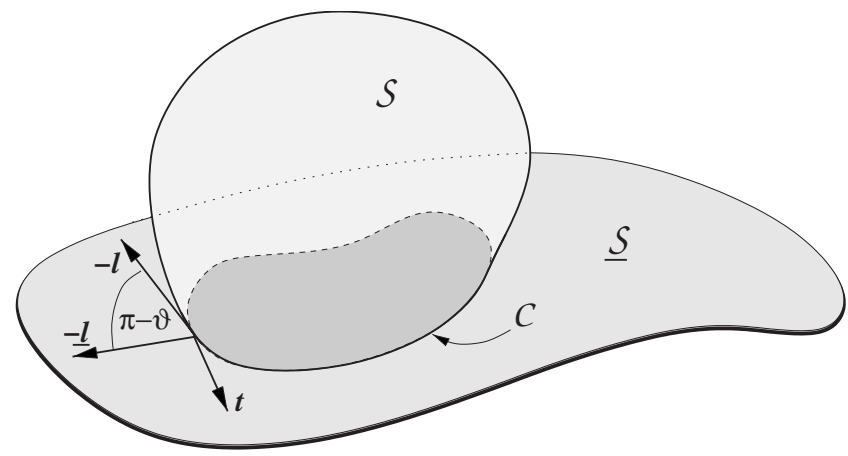

FIG. 1. Illustration of the geometry of surface adhension. Perpendicular to the contact line $\mathcal{C}$ two vectors exist, $\boldsymbol{l}$ and $\underline{\boldsymbol{l}}$, which are tangential to the surface $\mathcal{S}$ and substrate $\mathcal{S}$ respectively.

\section{MATHEMATICAL SETUP}

\section{A. Differential geometry}

In order to describe the adhering surfaces in a parametrization-free way, we use a covariant differential geometric language. Our notation is essentially standard and will follow the one used in Refs. [8-12]. Briefly, a surface $\mathcal{S}$ is described by the embedding function $\boldsymbol{X}\left(\xi^{1}, \xi^{2}\right) \in \mathbb{R}^{3}$, where the $\xi^{a}(a \in\{1,2\})$ are a suitable set of local coordinates on the surface. This induces two local tangent vectors $\boldsymbol{e}_{a}=\partial \boldsymbol{X} / \partial \xi^{a}$ and a normal vector satisfying $\boldsymbol{n} \cdot \boldsymbol{e}_{a}=0$ and $\boldsymbol{n}^{2}=1$. Furthermore, the two fundamental forms of the surface are needed: namely, (i) the metric tensor $g_{a b}=\boldsymbol{e}_{a} \cdot \boldsymbol{e}_{b}$ and (ii) the extrinsic curvature tensor $K_{a b}=\boldsymbol{e}_{a} \cdot \nabla_{b} \boldsymbol{n}=-\boldsymbol{n} \cdot \nabla_{a} \boldsymbol{e}_{b}$. The symbol $\nabla_{a}$ is the metric-compatible covariant derivative. The trace of the extrinsic curvature tensor will be denoted by $K=K_{a}^{a}=K_{a b} g^{a b}$, which for a sphere of radius $r$ with outward-pointing normal vector is positive and has the value $K=2 / r$. The determinant $K_{\mathrm{G}}=\operatorname{det}\left(K_{a}^{b}\right)$ is the Gaussian curvature. It can be written as half the Ricci scalar curvature $R=g^{a c} g^{b d} R_{a b c d}$, the double contraction of the Riemann tensor. This link between intrinsic and extrinsic curvatures is a consequence of the (doubly contracted) Gauss-Codazzi equation $R=K^{2}-K_{a b} K^{a b}$ $\left[=2 \operatorname{det}\left(K_{a}^{b}\right)\right]$. As usual, indices are lowered or raised with the metric or its inverse, respectively, and a repeated index $a$ (one up, one down) implies a summation over $a=1,2$. More background on differential geometry can be found in Refs. [13-15].

\section{B. Geometry at the contact line}

By "contact line" we will denote the curve $\mathcal{C}$ along which the surface detaches from the substrate. Its local direction is given by the tangent vector $\boldsymbol{t}=t^{a} \boldsymbol{e}_{a}$ (see Fig. 1), which is tangential to $\mathcal{C}$, the surface $\mathcal{S}$, and the substrate surface $\mathcal{S}$ (which itself might also be deformable). Here and in what follows (with the exception of Sec. IV B) we will use underlining in order to indicate quantities referring to the substrate.

Perpendicular to $\mathcal{C}$ we can define local normal vectors which are either tangential to $\mathcal{S}$ or $\mathcal{S}$ : namely, $\boldsymbol{l}=l^{a} \boldsymbol{e}_{a}$ and $\underline{\boldsymbol{l}}$ $=\underline{l}^{a} \underline{\boldsymbol{e}}_{a}$, respectively (see Fig. 1). Also, we will have two surface normals $\boldsymbol{n}$ and $\underline{\boldsymbol{n}}$. If the surface contacts the substrate smoothly at a contact angle of $180^{\circ}$, we will have $\boldsymbol{e}_{a}=\underline{\boldsymbol{e}}_{a}, \boldsymbol{n}$ $=\underline{\boldsymbol{n}}$, and $\boldsymbol{l}=\underline{\boldsymbol{l}}$ there; however, their derivatives perpendicular to the contact line need not coincide, since the curvatures of surface and substrate generally need not be identical. In fact, the values of perpendicular and parallel components of these curvatures (and possibly their higher derivatives) will be among the primary focus of this paper. They will be denoted by

$$
\begin{gathered}
K_{\perp}=l^{a} l^{b} K_{a b}=-\boldsymbol{n} \cdot \nabla_{\perp} \boldsymbol{l}, \\
K_{\|}=t^{a} t^{b} K_{a b}=-\boldsymbol{n} \cdot \nabla_{\|} \boldsymbol{t}, \\
K_{\perp \|}=K_{\| \perp}=l^{a} t^{b} K_{a b}=-\boldsymbol{n} \cdot \nabla_{\perp} \boldsymbol{t}=-\boldsymbol{n} \cdot \nabla_{\|} \boldsymbol{l},
\end{gathered}
$$

where we also introduced the two directional surface derivatives perpendicular and parallel to $\mathcal{C}$,

$$
\nabla_{\perp}=l^{a} \nabla_{a} \text { and } \nabla_{\|}=t^{a} \nabla_{a} .
$$

We analogously define $\underline{K}_{\perp}, \underline{K}_{\|}$, and $\underline{K}_{\perp \|}$.

\section{Hamiltonian}

In the present work we will exclusively study surfaces whose energy $H$ is given by a surface integral over a scalar energy density $\mathcal{H}$, which is constructed from the local surface geometry:

$$
H=\int_{\mathcal{S}} d A \mathcal{H}\left(g_{a b}, K_{a b}, \nabla_{a} K_{b c}, \ldots\right) .
$$

The integral extends over the entire surface $\mathcal{S}$, and the area element $d A$ is given by $\sqrt{g} d \xi^{1} d \xi^{2}$, where $g=\operatorname{det}\left(g_{a b}\right)$ is the metric determinant. For those parts of the surface which adhere to a substrate we will assume an additional adhesion energy density

$$
\mathcal{H}_{\text {adhesion }}=-w\left(\xi^{1}, \xi^{2}\right) \leqslant 0
$$

which may in general be a function of position.

\section{DETERMINING THE BOUNDARY CONDITIONS}

\section{A. Continuity considerations}

As we will see, the adhesion balance between surface and substrate will result in a discontinuous change of some surface property across the contact line. However, the form of the energy density restricts which quantities can be discontinuous, since it needs to remain integrable.

Most obviously, the shape itself has to be continuous. Yet already its first derivative may display a jump, as it does in the case of capillary adhesion. The energy density is given simply by

$$
\mathcal{H}_{\text {capillary }}=\sigma,
$$

and a kink in the surface at the contact line-i.e., a finite contact angle - is not associated with an extra energy.

For curvature elastic surfaces the situation is different. There, the energy density of the surface is given by the wellknown expression $[3,4]$ 


$$
\mathcal{H}_{\text {bend }}=\frac{1}{2} \kappa\left(K-K_{0}\right)^{2}+\frac{1}{2} \bar{\kappa} R,
$$

where $\kappa$ and $\bar{\kappa}$ are bending modulus and saddle-splay modulus, respectively, and $K_{0}$ describes a spontaneous curvature of the elastic surface. A kink in the surface at the contact line implies a $\delta$ singularity in the curvature, whose square is nonintegrable. Hence, the surface needs to be differentiable across the contact line, and the distinction between surface and substrate tangents and normals drawn in Sec. II B becomes unnecessary. Moreover, a quick glance at Eqs. (2b) and (2c) shows that both $K_{\|}$and the off-diagonal curvature $K_{\perp \|}$ are expressible as a tangential derivative along the curve $\mathcal{C}$ of a quantity continuous across $\mathcal{C}$; hence, both these curvatures will also be continuous. It is only the perpendicular curvature component $K_{\perp}$ which might possess a discontinuity, and indeed we will see that it does.

Finally, even higher-order derivatives might occur in surface Hamiltonians. For instance, Goetz and Helfrich have studied a curvature-gradient term of the form

$$
\mathcal{H}_{\text {grad }}=\frac{1}{2} \kappa_{\nabla}\left(\nabla_{a} K\right)\left(\nabla^{a} K\right) \equiv \frac{1}{2} \kappa_{\nabla}(\nabla K)^{2},
$$

which in a generalized higher-curvature Hamiltonian prevents the occurrence of infinitely sharp curvature changes [5]. In this case it is obvious that all curvature components have to be continuous along the substrate, since otherwise again a squared $\delta$ singularity results. Moreover, most of the first-order directional derivatives are automatically continuous: the parallel ones, $\nabla_{\|}$, again differentiate quantities along $\mathcal{C}$ which are continuous across $\mathcal{C}$ and thus are themselves continuous. For the perpendicular ones it turns out that $\nabla_{\perp} K_{\|}$ and $\nabla_{\perp} K_{\perp \|}$ are continuous, while $\nabla_{\perp} K_{\perp}$ is not. This is intuitively reasonable, since every term involving a "I") features at least one less derivative across the contact line and thus cannot jump. A rigorous proof is, however, a bit more involved. One may, for instance, proceed like this: start with the contracted Codazzi-Mainardi equation [13-15] $\nabla_{a} K^{a b}$ $-\nabla^{b} K=0$ and project onto $l_{b}$. By decomposing the resulting identity into the local $(\boldsymbol{l}, \boldsymbol{t})$ frame, it can be cast in the form

$$
\nabla_{\perp} K_{\|}=\nabla_{\|} K_{\perp \|}+\left(K_{\perp}-K_{\|}\right) \boldsymbol{t} \cdot \nabla_{\|} \boldsymbol{l}+2 K_{\perp \|} \boldsymbol{l} \cdot \nabla_{\perp} \boldsymbol{t} .
$$

Since every term on the right-hand side is continuous across $\mathcal{C}$ (recall that derivatives of tangent vectors are essentially curvatures), $\nabla_{\perp} K_{\|}$must be continuous as well. By projecting the contracted Codazzi-Mainardi equation on $t_{b}$ instead, one can show that $\nabla_{\perp} K_{\perp \|}$ is also continuous.

\section{B. Contact line variation}

For an adhering surface the total energy is stationary with respect to variations of the contact line along the substrate. Such a variation contributes twofold to the Hamiltonian: assume that locally the contact line is moved such that a bit of surface unbinds from the substrate. This removes its corresponding binding energy, as well as any elastic energy associated with the constraint of conforming to the substrate, and thus gives rise to an energy change $\delta H_{\text {bound. }}$ On the other hand, the unbound part of the surface acquires at the contact line a new boundary strip which implies also a change $\delta H_{\text {free }}$ in its elastic energy. The boundary condition at the contact line then follows from the stationarity condition

$$
\delta H_{\mathrm{cl}}=\delta H_{\text {bound }}+\delta H_{\text {free }}=0 .
$$

In the case of adhesion to a rigid substrate the bound contribution involves the variation along a surface of known shape. The corresponding term is thus conceptually very different from a deformable substrate or even the free variation, because in both these cases the local shape of the surface is not known. Below we will see how these differences manifest themselves when computing the boundary terms.

\section{Bound variation}

For definiteness, let the normal $\underline{\boldsymbol{l}}$ to the contact line $\mathcal{C}$ be directed towards the adhering portion of the surface (see Fig. 1). A local infinitesimal normal displacement $\varepsilon$ of the contact line along a rigid substrate thus implies the following obvious change in the bound part of the surface:

$$
\delta H_{\text {bound }}=-\int_{\mathcal{C}} d s(\underline{\mathcal{H}}-w) \varepsilon(s) .
$$

The underlining of $\mathcal{H}$ should again indicate that it is evaluated with geometric surface scalars (such as curvatures) pertaining to the substrate. If the substrate is flexible, the $w$ term remains, but the change in elastic energy will instead be taken care of by an additional free boundary variation.

\section{Free variation}

The change in energy due to the addition or removal of unbound parts to the boundaries of the surface is identical to the boundary terms in the variation of the free surface. In Ref. [10] it has been shown that for Hamiltonians up to curvature order these terms are given by [16]

$$
\delta H_{\text {free }}=-\int_{\mathcal{C}} d s l_{a}\left[\boldsymbol{f}^{a} \cdot \delta \boldsymbol{X}+\mathcal{H}^{a b} \boldsymbol{n} \cdot \delta \boldsymbol{e}_{b}\right] .
$$

Here, $f^{a}$ is the surface stress tensor, given by

$$
\boldsymbol{f}^{a}=\left(T^{a b}-\mathcal{H}^{a c} K_{c}^{b}\right) \boldsymbol{e}_{b}-\left(\nabla_{b} \mathcal{H}^{a b}\right) \boldsymbol{n},
$$

and we have also defined

$$
\mathcal{H}^{a b}=\frac{\delta \mathcal{H}}{\delta K_{a b}}
$$

and

$$
T^{a b}=-\frac{2}{\sqrt{g}} \frac{\delta(\sqrt{g} \mathcal{H})}{\delta g_{a b}} .
$$

Finally, $\delta \boldsymbol{X}$ and $\delta \boldsymbol{e}_{b}$ denote the change of contact line position and the associated change in the slope of the tangent vectors, respectively. Notice that the second term in Eq. (12) is only relevant if $\mathcal{H}^{a b} \neq 0$.

\section{SPECIFIC EXAMPLES}

In this section we will illustrate the above formalism by applying it to several important situations and surface Hamil- 
tonians. In Sec. IV A we first treat the problem of adhesion to a rigid substrate. We will see how known results (the Young-Dupré equation and the contact curvature condition for Helfrich membranes) follow with remarkable ease and can be extended just as quickly to new Hamiltonians. In Sec. IV B we look at the boundary conditions involving adhesion to deformable substrates. Specifically, in Sec. IV B 1 we look at the triple line between three tension surfaces, and in Sec. IV B 2 we study the adhesion of two vesicles.

A central ingredient in all this will be knowledge of the two tensors $\mathcal{H}^{a b}$ and $T^{a b}$ defined in Eqs. (14a) and (14b). While their determination is not particularly involved, these calculations have been performed previously by us [9-12] and we will thus simply reuse the results here.

\section{A. Adhesion to a rigid substrate}

Since the variation of the contact line has to proceed along the substrate, we must have

$$
\delta X=\varepsilon \underline{l} .
$$

No component in the $t$ direction is necessary, since for fluid surfaces this amounts to a reparametrization of $\mathcal{C}$. Notice that Eq. (15) is nothing but the Lie derivative of $\boldsymbol{X}$ along the substrate, since $\mathcal{L}_{\varepsilon \underline{l}} \boldsymbol{X}=\varepsilon \underline{\varepsilon}^{a} \nabla_{a} \boldsymbol{X}=\varepsilon \underline{l}^{a} \underline{e}_{a}=\varepsilon \underline{\boldsymbol{l}}$. This property holds generally, and we will make use of it later.

The normal component of the change in the surface tangent vectors $\boldsymbol{e}_{b}$ only contributes if $\mathcal{H}^{a b} \neq 0$-i.e., if curvature terms enter the Hamiltonian. We will assume that they do it in such a way that differentiability of the surfaces is implied (see Sec. III A), so that no distinction needs to be drawn between normal and tangent vectors of substrate and adhering surface. We then find

$$
\boldsymbol{n} \cdot \delta \boldsymbol{e}_{b}=\boldsymbol{n} \cdot \nabla_{b} \delta \boldsymbol{X}=\varepsilon l^{c} \boldsymbol{n} \cdot \nabla_{b} \underline{\boldsymbol{e}}_{c}=-\varepsilon l^{c} \underline{K}_{b c},
$$

where in the last step the equation of Weingarten [13-15] has been used; this is again the Lie derivative along the substrate [17]. Notice that there still remains a distinction between curvatures of substrate and surface; hence, the derivative of the tangent vectors resulting from a variation along the substrate yields the substrate curvature and not the free surface curvature.

\section{Capillary surfaces}

In this case the energy density is given by Eq. (6), and as we have seen in Sec. III A, we will expect a discontinuity in slope at $\mathcal{C}$. The bound variation is

$$
\delta H_{\text {bound }}=-\int_{\mathcal{C}} d s(\sigma-w) \varepsilon(s) .
$$

For this Hamiltonian we have [9-12] $\mathcal{H}^{a b}=0$ and $\boldsymbol{f}^{a}=$ $-\sigma g^{a b} \boldsymbol{e}_{b}=-\sigma \boldsymbol{e}^{a}$, and therefore

$$
l_{a} f^{a} \cdot \delta \boldsymbol{X}=-\sigma l_{a} \boldsymbol{e}^{a} \cdot \varepsilon \underline{\boldsymbol{l}}=-\sigma \varepsilon \boldsymbol{l} \cdot \underline{\boldsymbol{l}}=-\sigma \varepsilon \cos (\pi-\vartheta),
$$

where $\vartheta$ is the angle between capillary surface and substrate-in other words, the contact angle (see Fig. 1). Equation (10) thus specializes to

$$
\delta H_{\mathrm{cl}}=-\int_{\mathcal{C}} d s[\sigma-w+\sigma \cos \vartheta] \varepsilon(s) .
$$

Since $\varepsilon(s)$ is arbitrary, the term in square brackets must vanish, which gives the Young-Dupré equation (1).

\section{Helfrich Hamiltonian}

Let us now look at the energy density (7) which describes the continuum behavior of (tensionless) fluid lipid bilayers. Since $K$ appears quadratic, the surface will now be differentiable across the contact boundary, as pointed out in Sec. III A.

A simple argument shows that the intrinsic contribution $\frac{1}{2} \bar{\kappa} R=\bar{\kappa} K_{\mathrm{G}}$ will not matter for the boundary conditions. Since the contact line is varied locally, we can focus our attention on a membrane strip of finite width and fixed edges, covering the contact line. Due to the Gauss-Bonnet theorem [13-15], its contribution will not change upon variation of the contact line (which lies in the middle of the strip) and must therefore not enter any condition resulting from it. For readers not convinced by this quick argument, the following derivation shows explicitly (but less elegantly) that the $\bar{\kappa}$ contributions indeed drop out.

Let us begin with the stress tensor. From Refs. [9-12] we find

$$
l_{f} f^{a} \cdot l=-\frac{1}{2} \kappa\left(K-K_{0}\right)^{2}+\kappa\left(K-K_{0}\right) K_{\perp} .
$$

Notice that this expression is independent of $\bar{\kappa}$ : the Gaussian contribution in the Helfrich Hamiltonian does not create membrane stresses. Next, we look at the dependence of $\mathcal{H}$ on the extrinsic curvature. From Ref. [12] we get

$$
\mathcal{H}^{a b}=\kappa\left(K-K_{0}\right) g^{a b}+\bar{\kappa}\left(K g^{a b}-K^{a b}\right) .
$$

Here the $\bar{\kappa}$ contribution does not cancel right away. To obtain the necessary derivatives of the Ricci curvature, we exploited the identity $R=K^{2}-K_{a b} K^{a b}$. With the help of Eqs. (16) and (21) we can further calculate

$$
\begin{aligned}
l_{a} \mathcal{H}^{a b} \boldsymbol{n} \cdot \delta \boldsymbol{e}_{b} & =\left\{-\kappa\left(K-K_{0}\right) \underline{K}_{\perp}-\bar{\kappa}\left(K_{\|} \underline{K}_{\perp}-K_{\perp \|} \underline{K}_{\perp \|}\right)\right\} \varepsilon \\
& =-\left\{\kappa\left(K-K_{0}\right) \underline{K}_{\perp}+\frac{1}{2} \bar{\kappa} \underline{R}\right\} \varepsilon .
\end{aligned}
$$

The final step follows from the continuity conditions $\underline{K}_{\|}$ $=K_{\|}$and $\underline{K}_{\perp \|}=K_{\perp \|}$ from Sec. III A and the representation of the Ricci scalar curvature as the Gaussian curvature determinant $\frac{1}{2} \underline{R}=\underline{K}_{\mathrm{G}}=\underline{K}_{\perp} \underline{K}_{\|}-\underline{K}_{\perp \|}^{2}$.

Inserting Eqs. (20) and (22) into the general expression for the free variation, Eq. (12), and adding the bound variation (11), we arrive at the total energy change upon contact line variation: 


$$
\begin{aligned}
\delta H_{\mathrm{cl}}= & -\int_{\mathcal{C}} d s\left\{\frac{1}{2} \kappa\left(\underline{K}-K_{0}\right)^{2}+\frac{1}{2} \bar{\kappa} \underline{R}-w\right. \\
& -\frac{1}{2} \kappa\left(K-K_{0}\right)^{2}+\kappa\left(K-K_{0}\right) K_{\perp} \\
& \left.-\kappa\left(K-K_{0}\right) \underline{K}_{\perp}-\frac{1}{2} \bar{\kappa} \underline{R}\right\} \varepsilon(s) \\
= & -\int_{\mathcal{C}} d s\left\{\frac{1}{2} \kappa\left(K_{\perp}-\underline{K}_{\perp}\right)^{2}-w\right\} \varepsilon(s),
\end{aligned}
$$

where in the second step we again made use of the continuity condition $K_{\|}=\underline{K}_{\|}$. Notice that the $\bar{\kappa}$ dependence has finally canceled out completely, as we have argued above. Equation (23b) implies a discontinuity in the perpendicular curvature $K_{\perp}$ as the appropriate adhesion boundary condition:

$$
K_{\perp}-\underline{K}_{\perp}=\sqrt{\frac{2 w}{\kappa}} .
$$

The correct sign after taking the square root follows from the fact that the detaching surface must not penetrate the substrate; unfortunately, this depends on ones specific choice of the surface normal vectors.

Quite remarkably, this boundary condition depends neither on the spontaneous curvature $K_{0}$ (the contribution to the Hamiltonian linear in $K$ does not enter) nor on the local parallel curvature $K_{\|}$. It would also remain unaffected if the bilayer were under a finite tension $\sigma$. Formally, it is easily seen to cancel; physically, the reason is that the jump we would expect from the Young-Dupré equation (1) cannot materialize since the curvature terms in the energy density enforce differentiability of the profile at $\mathcal{C}$.

Equation (24) coincides with the result given previously in Ref. [8]. Its axisymmetric version was first quoted in Ref. [18], and its specialization to a straight contact line can be found in Ref. [19]. We want to stress that the $\mathcal{H}^{a b} \boldsymbol{n} \cdot \delta \boldsymbol{e}_{b}$ term in Eq. (12), which is responsible for the third line in Eq. (23a), was crucial in obtaining Eq. (24). Leaving it out-i.e., only treating the problem as a stress balance-will not result in the correct boundary condition, as first pointed out in Ref. [20]. The only exception (treated via stress-balance in Refs. $[8,21])$ is the special case of a flat substrate, in which case $\delta \boldsymbol{e}_{b} \equiv 0$ and the missing contribution vanishes anyway. The deeper reason for the apparent failure of a stress-based approach is the interdependence of the variations $\delta \boldsymbol{X}$ and $\delta \boldsymbol{e}_{b}$, as enforced by the rigid substrate. This point will become more clear once we have studied deformable substrates in Sec. IV B.

\section{General curvature Hamiltonians}

It is not difficult to extend the above analysis to the entire class of Hamiltonians which depend on intrinsic and extrinsic surface curvature. Since (i) for two-dimensional surfaces the Ricci scalar $R$ uniquely determines the Riemann tensor via $R_{a b c d}=\frac{1}{2} R\left(g_{a c} g_{b d}-g_{a d} g_{b c}\right)$ and since (ii) the CodazziMainardi equation permits the replacement of products of the extrinsic curvature tensor $K_{a b}$ with powers of the extrinsic curvature scalar $K$ and the Ricci scalar $R$, we can without loss of generality assume that any such curvature Hamiltonian has been brought into the form

$$
\mathcal{H}=\mathcal{H}(K, R)
$$

Once more using $R=K^{2}-K_{a b} K^{a b}$, we can derive the identities

$$
\begin{gathered}
\frac{\partial R}{\partial g_{a b}}=-2 R^{a b}=-R g^{a b}, \\
\frac{\partial R}{\partial K_{a b}}=2\left(K g^{a b}-K^{a b}\right) .
\end{gathered}
$$

Notice that the first equality in Eq. (26a) holds generally, while the second is specific for surfaces, since it uses the identity $R^{a b}=\frac{1}{2} \operatorname{Rg}^{a b}$ valid only in two dimensions.

We can now determine the two tensors $T^{a b}$ and $\mathcal{H}^{a b}$. Using the definitions (14) and the identities (26), a quick calculation leads to

$$
\begin{gathered}
T^{a b}=-\mathcal{H} g^{a b}+2 \frac{\partial \mathcal{H}}{\partial K} K^{a b}+2 \frac{\partial \mathcal{H}}{\partial R} R g^{a b}, \\
\mathcal{H}^{a b}=\frac{\partial \mathcal{H}}{\partial K} g^{a b}+2 \frac{\partial \mathcal{H}}{\partial R}\left(K g^{a b}-K^{a b}\right) .
\end{gathered}
$$

Before we proceed, let us look once more at the continuity requirements spelled out in Sec. III A. The question to be answered is whether our general Hamiltonian $\mathcal{H}$ enforces differentiability. If it does not involve the curvature at all, it is of the form (6) and will permit kinks, but this case has been treated in Sec. IV A 1. However, differentiability is not automatically guaranteed once curvature appears. In particular, kinks are possible if $\mathcal{H}$ is linear in $K$ or $R$, since both curvatures involve the component $K_{\perp}$ across the boundary only linearly. The resulting $\delta$ singularity is hence integrable and adds a finite extra contribution to the energy (and its variation) which we would need to consider separately. While such a situation can also be treated within the general framework presented in this paper, the details are surprisingly tricky. Since we prefer not to obfuscate the overall picture by devoting a disproportionate amount of space to these singular cases, we will from now on assume that differentiability holds. The reader should notice that this will be the case once a nonzero bending modulus $\kappa$ is present. Indeed, for dimensional consistency it is implausible to assume that a term linear in $R$ will occur without its "partner" quadratic in $K$. However, a Hamiltonian of the form $\sigma+\beta K$ is not irrelevant and occurs, for instance, if one asks what are the shapes for vesicles of given area and area difference (between outer and inner monolayer) which have maximal volume, as Svetina and Žekš have done [22]. The treatment of the adhesion balance for this case can be found in Ref. [8].

Assuming differentiability from now on, we can combine the expressions (27) for the tensors $T^{a b}$ and $\mathcal{H}^{a b}$ with the boundary variations in Eqs. (15) and (16) and from this readily find 


$$
\begin{aligned}
& l_{a} f^{a} \cdot \delta \boldsymbol{X}=-\varepsilon\left[\mathcal{H}-\frac{\partial \mathcal{H}}{\partial K} K_{\perp}-\frac{\partial \mathcal{H}}{\partial R} R\right], \\
& l_{a} \mathcal{H}^{a b} \boldsymbol{n} \cdot \delta \boldsymbol{e}_{b}=-\varepsilon\left[\frac{\partial \mathcal{H}}{\partial K} \underline{K}_{\perp}+\frac{\partial \mathcal{H}}{\partial R} \underline{R}\right] .
\end{aligned}
$$

The total contact line variation is therefore given by

$$
\begin{aligned}
\delta H_{\mathrm{cl}}= & -\int_{\mathcal{C}} d s\left\{\underline{\mathcal{H}}-w-\mathcal{H}+\frac{\partial \mathcal{H}}{\partial K} K_{\perp}+\frac{\partial \mathcal{H}}{\partial R} R\right. \\
& \left.-\frac{\partial \mathcal{H}}{\partial K} \underline{K}_{\perp}-\frac{\partial \mathcal{H}}{\partial R} R\right\} \varepsilon(s) .
\end{aligned}
$$

The requirement $\delta H_{\mathrm{cl}}=0$ now gives rise to the remarkably succinct boundary condition

$$
(\underline{\mathcal{H}}-\mathcal{H})-\frac{\partial \mathcal{H}}{\partial K}\left(\underline{K}_{\perp}-K_{\perp}\right)-\frac{\partial \mathcal{H}}{\partial R}(\underline{R}-R)=w .
$$

This equation generalizes the contact curvature condition (24) to arbitrary Hamiltonians of curvature order. Notice that despite its rather "systematic" appearance, this general condition is not symmetric between surface and substrate; i.e., it does not remain invariant when replacing underlined and not-underlined quantities, unlike the special case of the contact curvature condition (24) for Helfrich membranes. Also, the parallel curvature $K_{\|}$will generally enter the boundary condition, exposing the cancellation in the case of the Helfrich Hamiltonian as "accidental."

We can readily see how Eq. (30) reduces to the special cases we have treated above: for $\mathcal{H}=\frac{1}{2} \kappa K^{2}$ we have $\partial \mathcal{H} / \partial K=\kappa K$ and $\partial \mathcal{H} / \partial R=0$, such that the left-hand side of Eq. (30) becomes $\frac{1}{2} \kappa\left(\underline{K}^{2}-K^{2}\right)-\kappa K\left(\underline{K}_{\perp}-K_{\perp}\right)=\frac{1}{2} \kappa\left(\underline{K}_{\perp}-K_{\perp}\right)^{2}$, which leads to the contact curvature condition (24). And for a contribution $\frac{1}{2} \bar{\kappa} R$ in a Hamiltonian which enforces differentiability by the presence of other terms we have $\partial\left(\frac{1}{2} \bar{\kappa} R\right) / \partial K=0$ and $\partial\left(\frac{1}{2} \bar{\kappa} R\right) / \partial R=\frac{1}{2} \bar{\kappa}$, such that it cancels in the left-hand side of Eq. (30). We thereby see once more that the Gaussian curvature term in the Helfrich Hamiltonian (and thus the saddle splay modulus $\bar{\kappa}$ ) does not contribute to the boundary condition.

Let us conclude this section by casting a quick glance onto one more interesting special case: namely, the Hamiltonian

$$
\mathcal{H}=\frac{1}{2} \bar{\kappa}_{2} R^{2} .
$$

This quartic expression, which also enters the theory developed in Ref. [5], is the lowest-order non-trivial intrinsic curvature Hamiltonian one can write down for two-dimensional surfaces. Since $\partial \mathcal{H} / \partial K=0$ and $\partial \mathcal{H} / \partial R=\bar{\kappa}_{2} R$, we arrive at the (purely intrinsic) boundary condition

$$
R-\underline{R}=\sqrt{\frac{2 w}{\bar{\kappa}_{2}}},
$$

whose similarity with the usual contact curvature condition (24) is quite striking.

\section{General quartic Hamiltonian}

The Helfrich Hamiltonian terminates the expansion of the surface energy at the dimensional order length ${ }^{-2}$. For symmetric membranes the next order is the quartic one, length ${ }^{-4}$, and it adds four more terms to the energy [23]:

$$
\mathcal{H}_{4}=\frac{1}{4} \kappa_{4} K^{4}+\kappa_{\times} K^{2} R+\frac{1}{2} \bar{\kappa}_{2} R^{2}+\frac{1}{2} \kappa_{\nabla}(\nabla K)^{2} .
$$

While all four moduli have the same units-energy times length ${ }^{4}$ - they are not of the same order in surface derivatives. The first three terms are scalar functions of the surface curvatures-i.e., special cases of the Hamiltonian (25)—but the fourth term involves the derivative of the curvature and is therefore of third order in surface derivatives. This has significant implications for the boundary conditions holding at quartic order: while one might initially surmise that all quartic moduli (and possibly also the quadratic ones) enter in a presumably lengthy equation, the continuity considerations from Sec. III A imply that all curvatures are continuous across the contact line due to the occurrence of the gradient$K$ term in (33). In consequence, none of the undifferentiated curvature terms influences the boundary condition, which is exclusively determined by the gradient term.

We have thus seen that it suffices in quartic order to study the implications of curvature gradients alone-i.e., the Hamiltonian (8). From earlier work $[11,12]$ we know that in this case

$$
\begin{gathered}
l_{f} f^{a} \cdot \boldsymbol{l}=\kappa_{\nabla}\left[\left(\nabla_{\perp} K\right)^{2}-\frac{1}{2}(\nabla K)^{2}-K_{\perp} \Delta K\right], \\
\mathcal{H}^{a b}=-\kappa_{\nabla}(\Delta K) g^{a b} .
\end{gathered}
$$

However, simplemindedly inserting these expressions into the formulas we have used so far does not give the correct result. Here is why: when calculating $\mathcal{H}^{a b}$ and $T^{a b}$ to obtain Eqs. (34), we varied the Hamiltonian (8) with respect to $K_{a b}$ and $g_{a b}$, respectively, and identified the bulk terms (see Sec. IV in the Appendix of Ref. [12]). Yet these variations also leave boundary terms, since the curvature $K=g^{a b} K_{a b}$ appears differentiated. For the purpose of identifying $\mathcal{H}^{a b}$ and $T^{a b}$ they are irrelevant, but they evidently matter now that we are interested in the total energy change upon displacing the boundary. Moreover, within the variational framework introduced in Ref. [10], on which the present treatment relies, the $\delta K_{a b}$ and $\delta g_{a b}$ variations are indeed independent from the $\delta \boldsymbol{X}$ and $\delta \boldsymbol{e}_{a}$ terms already included in Eq. (12), so they can be simply added to this expression. If we go through the calculation, we see that the two tensor variations can be combined nicely into a single scalar one, since they occur in the combination $g^{a b} \delta K_{a b}+K_{a b} \delta g^{a b}=\delta K$, and we end up with the additional boundary contribution

$$
\delta H_{\text {free, grad }}=\kappa_{\nabla} \int_{\mathcal{C}} d s l_{a}\left(\nabla^{a} K\right) \delta K .
$$

This is of course exactly the boundary term we would expect for the variation of a Hamiltonian density whose functional 
form is the square of the gradient of a scalar, so everything is consistent.

To evaluate the right hand side of Eq. (35), note that the variation is once more given by the Lie derivative along the substrate. Since $K$ is a scalar, we obtain the simple expression

$$
\delta K=\mathcal{L}_{\varepsilon l} K=\varepsilon l^{a} \nabla_{a} \underline{K}=\varepsilon \nabla_{\perp} \underline{K} .
$$

Together with Eqs. (34) we then obtain the total contact line variation as

$$
\begin{aligned}
\delta H_{\mathrm{cl}}= & -\int_{\mathcal{C}} d s\left\{\frac{1}{2} \kappa_{\nabla}(\nabla \underline{K})^{2}-w\right. \\
& +\kappa_{\nabla}\left[\left(\nabla_{\perp} K\right)^{2}-\frac{1}{2}(\nabla K)^{2}-K_{\perp} \Delta K\right] \\
& \left.+\kappa_{\nabla}(\Delta K) \underline{K}_{\perp}-\kappa_{\nabla}\left(\nabla_{\perp} K\right)\left(\nabla_{\perp} \underline{K}\right)\right\} \varepsilon(s) \\
= & -\int_{\mathcal{C}} d s\left\{\frac{1}{2} \kappa_{\nabla}\left(\nabla_{\perp} K_{\perp}-\nabla_{\perp} \underline{K}_{\perp}\right)^{2}-w\right\} \varepsilon(s)
\end{aligned}
$$

where in the last step we used the continuity of curvatures and their $\nabla_{\|}$derivatives (as discussed in Sec. III A) as well as the decomposition $(\nabla K)^{2}=\left(\nabla_{\perp} K\right)^{2}+\left(\nabla_{\|} K\right)^{2}$. The boundary condition following from this specifies a jump in the perpendicular derivative of the perpendicular curvature:

$$
\nabla_{\perp}\left(K_{\perp}-\underline{K}_{\perp}\right)=\sqrt{\frac{2 w}{\kappa_{\nabla}}} .
$$

As remarked above, this constitutes the appropriate boundary condition for a curvature elastic theory including all terms up to quartic order. Its similarity with Eqs. (24) and (32) is again very striking, and one might surmise a pattern that would be followed by even higher-derivative theories. Notice, however, that the terms entering the derivation of Eq. (38) are quite different and that the additional term stemming from Eq. (35), which is absent in the simple curvature square case, is essential.

\section{B. Adhesion to deformable surfaces}

Compared to the previous section, there are two key differences if the substrate is not rigid. First, the absence of a known substrate shape along which a certain amount of deformation energy is to be paid removes the term involving $\underline{\mathcal{H}}$ in the bound variation (11). Second, for the same reason the contact line variation is no longer restricted to proceed along a substrate and will thus be of the more general form

$$
\delta \boldsymbol{X}=\varepsilon_{\perp} l+\varepsilon_{n} \boldsymbol{n} .
$$

The corresponding tangent vector variation, which occurs if $\mathcal{H}^{a b} \neq 0$, then leaves a term

$$
\boldsymbol{n} \cdot \delta \boldsymbol{e}_{b}=\boldsymbol{n} \cdot \nabla_{b}\left(\varepsilon_{\perp} \boldsymbol{l}+\varepsilon_{n} \boldsymbol{n}\right)=-\underline{K}_{b c} c^{c} \varepsilon_{\perp}+\nabla_{b} \varepsilon_{n} .
$$

Note that $\underline{K}_{b c}$ plays a different role here than previously. It no longer describes the curvature of the evidently nonexistent

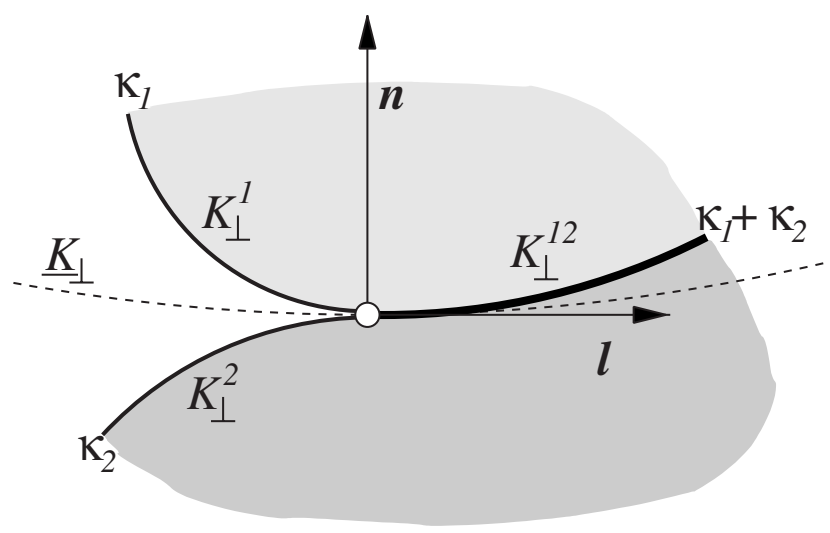

FIG. 2. Illustration of the geometry at the contact line between two adhering vesicles.

substrate. Rather, the tangential variation may proceed locally along a fictitious surface which is tangential to the other three surfaces that meet at the contact line. Encoding the higher-order derivative information necessary here, $\underline{K}_{b c}$ describes the curvature of that fictitious surface and $\underline{K}_{\perp}$ is the component perpendicular to $\mathcal{C}$ (see Fig. 2). This surface is of course not unique, and thus $\underline{K}_{\perp}$ is arbitrary-just as the two variations $\varepsilon_{\perp}$ and $\varepsilon_{n}$ themselves are.

\section{Three-phase capillary equilibrium}

The simplest example of a three-phase line between deformable surfaces occurs when three capillary interfaces meet-for instance, at the three-phase line between three mutually immiscible fluids 1,2 , and 3 , having mutual surface tensions $\sigma^{12}, \sigma^{23}$, and $\sigma^{31}$. In this case no adhesion energy is involved (or, alternatively, it may be considered as part of the surface tension). The contact line variation thus consists of three identical boundary variations

$$
\begin{aligned}
\delta H_{\mathrm{cl}} & =-\int d s\left\{l_{a}^{12} \boldsymbol{f}^{12 a}+l_{a}^{23} \boldsymbol{f}^{23 a}+l_{a}^{31} \boldsymbol{f}^{31 a}\right\} \delta \boldsymbol{X} \\
& =\int d s\left\{\sigma^{12} \boldsymbol{l}^{12}+\sigma^{23} \boldsymbol{l}^{23}+\sigma^{31} \boldsymbol{l}^{31}\right\} \delta \boldsymbol{X},
\end{aligned}
$$

from which we immediately find the boundary condition

$$
\boldsymbol{\sigma}^{12}+\boldsymbol{\sigma}^{23}+\boldsymbol{\sigma}^{31}=0
$$

This expresses nothing but the force balance between the three directional line tensions $\boldsymbol{\sigma}^{12}=\sigma^{12} \boldsymbol{l}^{12}$, etc., and is known as the Neumann triangle [1]. The vector equation (42) corresponds to two scalar equations (since there is no component along $t$ ). These are sufficient to determine the three contact angles between the three phases (because their sum equals $360^{\circ}$ ). Notice that this conversely implies that by measuring these angles one can only determine the ratios between the three tensions, not absolute values. How all this information is conveniently extracted is discussed in detail in Chap. 8 of Ref. [1]. 


\section{Adhesion of two vesicles}

For the case of two adhering vesicles we assume that vesicle 1 has bending modulus $\kappa_{1}$ and tension $\sigma_{1}$, while vesicle 2 has corresponding values $\kappa_{2}$ and $\sigma_{2}$. If the two bilayers can slide past each other in the region where they adhere, their joint bending modulus is given by $\kappa_{12}=\kappa_{1}+\kappa_{2}$, because the energies required to bend either one just add; the same applies to the tension: $\sigma_{12}=\sigma_{1}+\sigma_{2}$. We will for simplicity look at the case where the spontaneous curvature is zero. The contact line variation now contains one adhesion term and three free boundary variations. Using the decomposition of $\delta \boldsymbol{X}$ and $\delta \boldsymbol{e}_{b}$ as given in Eqs. (39) and (40), respectively, we find the total energy change to be

$$
\begin{aligned}
\delta H_{\mathrm{cl}}= & -\int d s\left\{\left[w-\frac{1}{2}\left(\kappa_{1}+\kappa_{2}\right)\left(K_{\perp}^{12}\right)^{2}\right.\right. \\
& \left.+\frac{1}{2} \kappa_{1}\left(K_{\perp}^{1}\right)^{2}+\frac{1}{2} \kappa_{2}\left(K_{\perp}^{2}\right)^{2}\right] \varepsilon_{\perp} \\
& -\nabla_{\perp}\left[\kappa_{1} K_{\perp}^{1}+\kappa_{2} K_{\perp}^{2}-\left(\kappa_{1}+\kappa_{2}\right) K_{\perp}^{12}\right] \varepsilon_{n} \\
& -\left[\kappa_{1} K_{\perp}^{1}+\kappa_{2} K_{\perp}^{2}-\left(\kappa_{1}+\kappa_{2}\right) K_{\perp}^{12}\right] \underline{K}_{\perp} \varepsilon_{\perp} \\
& \left.+\left[\kappa_{1} K_{\perp}^{1}+\kappa_{2} K_{\perp}^{2}-\left(\kappa_{1}+\kappa_{2}\right) K_{\perp}^{12}\right] \nabla_{\perp} \varepsilon_{n}\right\} .
\end{aligned}
$$

All corresponding $K_{\|}$contributions cancel, since $K_{\|}$is again continuous across $\mathcal{C}$; the same happens to the tensions. The four terms belonging to the independent variations $\varepsilon_{\perp}, \varepsilon_{n}$, $K_{\perp} \varepsilon_{\perp}$, and $\nabla_{\perp} \varepsilon_{n}$ must vanish individually. Notice that the last two have identical prefactors; in fact, using Eq. (40) as well as the obvious identities $\varepsilon_{\perp}=\boldsymbol{l} \cdot \boldsymbol{\delta} \boldsymbol{X}$ and $\varepsilon_{n}=\boldsymbol{n} \cdot \boldsymbol{\delta} \boldsymbol{X}$, we can rewrite the total variation (43) in the more transparent form [24]

$$
\begin{aligned}
\delta H_{\mathrm{cl}}= & -\int d s\left\{\left[-w-\frac{1}{2}\left(\kappa_{1}+\kappa_{2}\right)\left(K_{\perp}^{12}\right)^{2}\right.\right. \\
& \left.+\frac{1}{2} \kappa_{1}\left(K_{\perp}^{1}\right)^{2}+\frac{1}{2} \kappa_{2}\left(K_{\perp}^{2}\right)^{2}\right] \boldsymbol{l} \cdot \delta \boldsymbol{X} \\
& -\nabla_{\perp}\left[\kappa_{1} K_{\perp}^{1}+\kappa_{2} K_{\perp}^{2}-\left(\kappa_{1}+\kappa_{2}\right) K_{\perp}^{12}\right] \boldsymbol{n} \cdot \delta \boldsymbol{X} \\
& \left.-\left[\kappa_{1} K_{\perp}^{1}+\kappa_{2} K_{\perp}^{2}-\left(\kappa_{1}+\kappa_{2}\right) K_{\perp}^{12}\right] \boldsymbol{l} \cdot \delta \boldsymbol{n}\right\} .
\end{aligned}
$$

This identifies clearly the three independent variations which matter: one tangential and one perpendicular translation, described by $\boldsymbol{l} \cdot \delta \boldsymbol{X}$ and $\boldsymbol{n} \cdot \boldsymbol{\delta} \boldsymbol{X}$, respectively, and one rotation around the local contact line, specified by the variation $\boldsymbol{l} \cdot \delta \boldsymbol{n}$. The corresponding three boundary conditions are then given by

$$
\begin{gathered}
\kappa_{1}\left(K_{\perp}^{1}\right)^{2}+\kappa_{2}\left(K_{\perp}^{2}\right)^{2}-\left(\kappa_{1}+\kappa_{2}\right)\left(K_{\perp}^{12}\right)^{2}=2 w, \\
\nabla_{\perp}\left(\kappa_{1} K_{\perp}^{1}+\kappa_{2} K_{\perp}^{2}-\left(\kappa_{1}+\kappa_{2}\right) K_{\perp}^{12}\right)=0 \\
\kappa_{1} K_{\perp}^{1}+\kappa_{2} K_{\perp}^{2}-\left(\kappa_{1}+\kappa_{2}\right) K_{\perp}^{12}=0 .
\end{gathered}
$$

Our identification of the independent variations also permits an easy interpretation of these three conditions: the first two equations are an expression of a local stress balance (namely, of tangential and perpendicular forces). The third condition expresses the balance of torques around the contact line, a suspicion confirmed by the general form of the membrane torque tensor $[9,21,25]$.

Contrary to the case of vesicle adhesion to a rigid substrate, Eq. (24), these conditions also contain one which involves the derivative of curvatures: namely, Eq. (45b). Its origin is the perpendicular variation $\boldsymbol{n} \cdot \boldsymbol{\delta} \boldsymbol{X}$, forbidden if the substrate cannot move, and since this term multiplies the normal component of the stress tensor, which [as Eq. (13) informs us] always contains one more derivative than the tangential one, this brings about the higher-derivative condition.

Not surprisingly, the boundary conditions (45) look distinctly different from the contact curvature condition which holds for the adhesion of a single Helfrich membrane to a rigid substrate, Eq. (24). However, it is possible to rewrite them in such a way that the relation becomes more visible. The tangential stress balance (45a) and the torque balance (45c) can be combined to yield the symmetric equations

$$
\begin{aligned}
& \left(1+\frac{\kappa_{1}}{\kappa_{2}}\right)\left(K_{\perp}^{1}-K_{\perp}^{12}\right)^{2}=\frac{2 w}{\kappa_{1}}, \\
& \left(1+\frac{\kappa_{2}}{\kappa_{1}}\right)\left(K_{\perp}^{2}-K_{\perp}^{12}\right)^{2}=\frac{2 w}{\kappa_{2}} .
\end{aligned}
$$

From Eq. (45c) it follows that one of the $K_{\perp}^{i}$ is larger and the other one smaller than $K_{\perp}^{12}$. Hence, when taking the square root in Eqs. (46), exactly one of the two will necessitate a minus sign.

Let us look at two special cases of these boundary conditions which turn out to be quite instructive. First, if $\kappa_{2} \rightarrow \infty$, the second vesicle approaches the limit of a rigid substrate. In this case Eq. (46b) shows that $K_{\perp}^{12}=K_{\perp}^{2}$ and Eq. (46a) reduces to the old contact curvature condition we had just derived for rigid substrates, Eq. (24). And the curvature of this effective substrate is determined from $\nabla_{\perp}\left(K_{\perp}^{2}-K_{\perp}^{12}\right)=0$. This latter condition shows that the "substrate" curvature is even differentiable across $\mathcal{C}$-or, in other words, the "substrate" shape is a 3 times continuously differentiable function.

And second, if the two membranes have identical bending moduli $\kappa_{1}=\kappa_{2}=\kappa$, a "symmetrized" contact curvature condition ensues which reads

$$
\left(K_{\perp}^{1}-K_{\perp}^{12}\right)^{2}=\left(K_{\perp}^{2}-K_{\perp}^{12}\right)^{2}=\frac{w}{\kappa},
$$

which tells us that the (squared) curvature jump $2 w / \kappa$ demanded by the rigid substrate version (24) is shared in equal parts between the two membranes, while the final condition of perpendicular force balance becomes $\nabla_{\perp}\left(K_{\perp}^{1}+K_{\perp}^{2}\right.$ $\left.-2 K_{\perp}^{12}\right)=0$. The specialization of Eq. (47) to the adhesion between two vesicles of equal bending modulus in an axisymmetric configuration has previously been derived by Derganc et al. [26].

To conclude this section, let us take a final look at stresses and torques. Their balance has emerged with remarkable directness as the necessary equilibrium condition for the case 
of adhering vesicles. Why is this so different from the contact curvature condition at a rigid substrate, where a simple stress balance fails? Looking at Eq. (23a), we see indeed stress and torque contributions entering. Or even more generally, the term involving $\mathcal{H}^{a b} \boldsymbol{n} \cdot \boldsymbol{\delta} \boldsymbol{e}_{b}$ in Eq. (12) is the origin for the intrinsic torque, since it is sensitive to local rotations of the surface normal $[9,25]$. Yet for the case of rigid substrates rotations are entirely "enslaved" to translations, since the rotation of the tangent vectors must follow the local substrate curvature-see Eq. (16). Consequently, stresses and torques enter a single balance condition and cannot be disentangled. How their contributions conspire to create a single combined equilibrium is probably easiest traced back on the detour via the two-vesicle case: as we have seen above, tangential stress and torque balance together are responsible for the symmetrized equations (46), whose rigid substrate limit $\kappa_{2} \rightarrow \infty$ then yields the entangled balance condition (24).

\section{SUMMARY}

We have shown how the boundary conditions pertaining to the contact line between a fluid surface adhering to a solid substrate or another deformable surface can be extracted from a systematic boundary variation in a completely parametrization-independent way. We would like to close with a summary of our main results and some remarks.

(i) Integrability of the surface energy density $\mathcal{H}$ enforces continuity of certain geometric variables across the contact line.

(ii) The highest derivative in $\mathcal{H}$ thus dictates which geometric variables may change discontinuously across $\mathcal{C}$ in response to adhesion. Hence, for the Helfrich Hamiltonian the tension $\sigma$ does not enter the boundary condition even if it enters $\mathcal{H}$; likewise, neither tension $\sigma$ nor bending modulus $\kappa$ enters the boundary condition if also a gradient-curvaturesquared term is present in $\mathcal{H}$.

(iii) Higher-order derivatives in $\mathcal{H}$ create boundary terms in the variation which pick up surface variations that are one order lower. If the curvature enters $\mathcal{H}$, then a change in slope is noticed; if a gradient in curvature enters $\mathcal{H}$, then changes in curvature are noticed. For this reason the capillary Hamiltonian is the only one which only picks up translations, such that the energy minimization can be reinterpreted as a force balance. In all other cases higher-derivative deformations (such as torques or even more complicated constructs) contribute to the boundary variation.

(iv) More formally, the presence of a rigid substrate enslaves all higher-order variations (such as rotations or curvature changes) to the contact line translation and thus mixes their corresponding generalized stress contributions (such as torques) into a single balance equation. Under these conditions a simple stress balance must fail.

(v) The boundary conditions studied in this paper are local in nature and depend only on the highest-derivative term in the Hamiltonian. Yet lower-order terms may affect the surface shape in the proximity of the contact line, more precisely on length scales where these terms dominate the energy density, as previously pointed out by Seifert and Lipowsky [18]. For instance, Helfrich membranes under tension exhibit the characteristic length scale $\lambda=\sqrt{\kappa / \sigma}$, which signifies the crossover between small-scale bending domination and the large-scale tension regime. Hence, adhesion of a vesicle large compared to $\lambda$ may be characterized on scales larger than $\lambda$ by an effective contact angle determined from the Young-Dupré equation (1), even though upon closer inspection the membrane really adheres smoothly, obeying the contact curvature condition (24). Likewise, a quartic term such as the one from the Hamiltonian (8) is only expected to influence the neighborhood of the contact line within a proximity region $\lambda^{\prime}=\sqrt{\kappa_{\nabla} / \kappa}$.

(vi) Notice that the previous remark demands a subtle consistency check: for the presented framework to be applicable, the proximity length associated with the highest-order term dictating the nature of the boundary condition must still be larger than the length scale characterizing the finite range of the adhesion potential between the surfaces, which we in this treatment have from the outset assumed to be zero. In other words, the notion of a contact interaction would be inconsistent if it implies shape features on scales smaller than the actual potential range.

(vii) Generalizations to surfaces hosting additional scalar or vector fields (such as composition or tilt order) appear straightforward, since these are readily incorporated into the present framework [12].

(viii) To be sure, knowing the boundary conditions does not mean that one also knows the position of the contact line. Rather, the latter has to be determined simultaneously with the surface shape. In general this task is very difficult, but it is not the subject of the present work.

\section{ACKNOWLEDGMENTS}

We acknowledge the hospitality of IPAM, where this work was originally conceived. M.D. is grateful for the hospitality of UNAM, where it was completed, as well as for Emmy Noether Grant No. De775/1-3 by the German Science Foundation. J.G. acknowledges partial support from CONACyT Grant No. 51111 as well as DGAPA PAPIIT Grant No. IN119206-3.
[1] J. S. Rowlinson and B. Widom, Molecular Theory of Capillarity (Dover, New York, 2002).

[2] P. G. de Gennes, F. Brochard-Wyart, and D. Quere, Capillarity and Wetting Phenomena (Springer, Berlin, 2003).
[3] P. B. Canham, J. Theor. Biol. 26, 61 (1970).

[4] W. Helfrich, Z. Naturforsch. C 28, 693 (1973).

[5] R. Goetz and W. Helfrich, J. Phys. II 6, 215 (1996).

[6] This is true for droplets small compared to the capillary length 
$\ell=\sqrt{\sigma / \rho g}$, where $\sigma$ and $\rho$ are surface tension and density of the liquid, and $g$ is the Earth's acceleration. For instance, water under ambient conditions has $\ell \simeq 2.6 \mathrm{~mm}$.

[7] Notice that in the literature on "wetting" the tendency of a liquid to wet a substrate is usually not characterized by the adhesion energy, $w$ but rather by the spreading parameter $S$ $=w-2 \sigma$, which measures the difference in surface energy between the dry and the wet substrate. In terms of $S$ the YoungDupré equation reads $S / \sigma=\cos \vartheta-1$.

[8] R. Capovilla and J. Guven, Phys. Rev. E 66, 041604 (2002).

[9] R. Capovilla and J. Guven, J. Phys. A 35, 6233 (2002).

[10] J. Guven, J. Phys. A 37, L313 (2004).

[11] M. M. Müller, M. Deserno, and J. Guven, Europhys. Lett. 69, 482 (2005).

[12] M. M. Müller, M. Deserno, and J. Guven, Phys. Rev. E 72, 061407 (2005).

[13] M. Do Carmo, Differential Geometry of Curves and Surfaces (Prentice Hall, Englewood Cliffs, NJ, 1976).

[14] M. Spivak, A Comprehensive Introduction to Differential Geometry, 2nd ed. (Publish or Perish, Boston, 1979), Vol. 4.

[15] E. Kreyszig, Differential Geometry (Dover, New York, 1991).

[16] Equation (12) is written slightly differently in Ref. [10]. That the two forms are identical may be seen from the fact that since $\boldsymbol{e}_{b} \cdot \boldsymbol{n}=0$, we have $\boldsymbol{e}_{b} \cdot \delta \boldsymbol{n}=-\boldsymbol{n} \cdot \delta \boldsymbol{e}_{b}$.

[17] This is most easily seen in the following way: $\boldsymbol{n} \cdot \boldsymbol{\delta} \boldsymbol{e}_{b}=$ $-\boldsymbol{e}_{b} \cdot \delta \boldsymbol{n}=-\boldsymbol{e}_{b} \cdot \mathcal{L}_{\varepsilon l} \boldsymbol{n}=-\boldsymbol{e}_{b} \cdot \varepsilon l^{c} \nabla_{c} \boldsymbol{n}=-\boldsymbol{e}_{b} \cdot \varepsilon l^{c} \underline{K}_{c}^{a} \boldsymbol{e}_{a}=-\varepsilon l^{c} g_{a b} \underline{K}_{c}^{a}=$ $-\varepsilon l^{c} \underline{K}_{b c}$, which is exactly the right-hand side of Eq. (19).

[18] U. Seifert and R. Lipowsky, Phys. Rev. A 42, 4768 (1990).

[19] L. D. Landau and E. M. Lifshitz, Theory of Elasticity, 3rd ed. (Butterworth Heinemann, Oxford, 1986), Sec. 12, problem 6.

[20] M. M. Müller, Diploma thesis, MPI for Polymer Research and University of Mainz, 2004 available as document 284971 under http://edoc.mpg.de.

[21] J.-B. Fournier, Soft Matter 7, 883 (2007).

[22] S. Svetina and B. Žekš, Eur. Biophys. J. 17, 101 (1989).

[23] R. Capovilla, J. Guven, and J. A. Santiago, J. Phys. A 36, 6281 (2003).

[24] Notice once more that since $\boldsymbol{n} \cdot \boldsymbol{e}_{b}=\boldsymbol{n} \cdot \boldsymbol{l}=0$, we have $l^{b} \boldsymbol{n} \cdot \delta \boldsymbol{e}_{b}$ $=\boldsymbol{n} \cdot \delta\left(l^{b} \boldsymbol{e}_{b}\right)=\boldsymbol{n} \cdot \delta \boldsymbol{l}=\boldsymbol{- l} \cdot \delta \boldsymbol{n}$.

[25] M. M. Müller, M. Deserno, and J. Guven Phys. Rev. E 76, 011921 (2007).

[26] J. Derganc, B. Božič, S. Svetina, and B. Žekš, Biophys. J. 84, 1486 (2003). 